\title{
Immunocytochemical Study of Microvilli in a Gastric Carcinoma-derived Cell Line
}

\author{
Masako Kasami ${ }^{*}$, Tadayoshi Uezato ${ }^{2 \dagger}$, Yi-Xin Wu ${ }^{2}$, Yasuhisa Naito ${ }^{1}$, Hidehiko Ohta ${ }^{3}$, Michiya \\ Fujita $^{2}$, and Isamu Kino ${ }^{1}$ \\ ${ }^{1}$ Department of Pathology and ${ }^{2}$ Department of Biochemistry, Hamamatsu University, School of Medicine, 3600 \\ Handa, Hamamatsu 431-31 Japan, ${ }^{3}$ Kyorin University, Hachiouji 192 Japan
}

Key words: Microvilli/villin/ezrin/KATO-III cell

\begin{abstract}
$A B S T R A C T$. To study the structural components of microvilli of the KATO-III cell, we used anti-villin, -ezrin, and anti-MVM (microvillous membrane prepared against mouse intestinal microvilli) antibodies. Villin and ezrin cross-link actin bundles of microvilli such as those in the small intestine and renal proximal cells. Electromicroscopically, the cytoskeletal core of microvilli of the KATO-III cell was constituted of actin-filament bundles. The anti-villin antibody but not anti-ezrin antibody reacted with the KATO-III cell as demonstrated by FITC-immunofluorescence and PAP-staining. Anti-villin, anti-MVM, but not anti-ezrin antibody, reacted with the KATO-III cell surface and with intracellular materials. Western blot analysis using anti-villin and anti-MVM antibodies revealed proteins of $\sim 95 \mathrm{kDa}$ (villin), and $15 \mathrm{kDa}$ in the microsomal membrane fractions of KATOIII cells, respectively. Immunocytochemical and confocal laser microscopic studies showed that the cell-surface and the intracellular microcysts of KATO-III cells were preferentially decorated by anti-villin and anti-MVM antibodies. These data suggested that some actin-binding proteins, such as villin, were localized at the cell surface and on some of the intracellular cytoplasmic structures of the KATO-III cell.
\end{abstract}

Sporadic microvilli occur on the cell surface of divergent types of epithelia. The occurrence of an elaborate structure defined as a brush border, the major part of which is formed by innumerable microvilli, is limited to certain types of epithelial cells such as intestinal mucosal absorptive cells and renal tubular re-absorptive cells. These microvilli are made up of a membrane element and a lining cytoskeletal structure. Of the cytoskeletal components, villin is the best characterized protein (5). Tumor cells of the majority of intestinal and renal carcinomas stained positively for villin which located mostly in the microvilli $(8,12,15,17,18,19)$. Villin (7), ezrin (3) and fimbrin (1) are known as cross-linkers of Factin filaments in the core bundle of a microvillus. In the present work we characterized microvilli and the brush border-like structure observed in the intracellular microcysts of KATO-III cells (22), a cell line established from an undifferentiated type of gastric carcinoma (20).

\footnotetext{
$\dagger$ To whom correspondence should be addressed.

* Present address: Department of Pathology, Vanderbilt University, School of Medicine, Nashville, TN37232 U.S.A.

Abbreviations: PAP; Peroxidase anti-peroxidase, PMSF; Phenylmethylsulfonyl fluoride, NEM; N-Ethylmaleimide.
}

\section{MATERIALS AND METHODS}

Cells. The human gastric carcinoma cell line KATO-III used in this study was originally derived from a metastatic pleural effusion obtained from a 55-year-old male patient with scirrhous carcinoma of the stomach (20). Cells were cultured at $37^{\circ} \mathrm{C}$ under $5 \% \mathrm{CO}_{2}$ in air for 7 days and then collected by centrifugation at $800 \times \mathrm{g}$ for $5 \mathrm{~min}$.

Antibodies and Immunocytochemical staining. Cells were fixed with methacarn $(60 \%$ methanol, $30 \%$ chloroform and $10 \%$ acetic acid) (2) and then embedded in paraffin. The $3 \mu \mathrm{m}$ sections of the cells were incubated with the primary antibodies to villin (Immunotech S.A.), ezrin (Chemicon International Inc.) and MVM (prepared in this study) at a dilution of $1 / 100-1 / 200$ in PBS, $\mathrm{pH} 7.4$, containing 1\% BSA for 1 hour. FITC-labeled goat anti-rabbit IgG, or anti-mouse IgG (Cappel, 1/100) was then incubated with the sections for $45 \mathrm{~min}$. The sections were examined with fluorescence microscope (Olympus Co. RFL) or with a laser confocal microscope (MR600 , Bio-Rad). Anti-MVM antibody was prepared using mouse intestinal brush border membranes as described previously (12).

Transmission electron microscopy. The cells were suspended in $0.1 \mathrm{M}$ cacodylate buffer, $\mathrm{pH} 7.4$, containing $1 \% \mathrm{glu}$ taraldehyde and allowed to stand at $20^{\circ} \mathrm{C}$ for $15 \mathrm{~min}$. The suspension was centrifuged for $5 \mathrm{~min}$ at $800 \times \mathrm{g}$ and washed three 
M. Kasami et al.
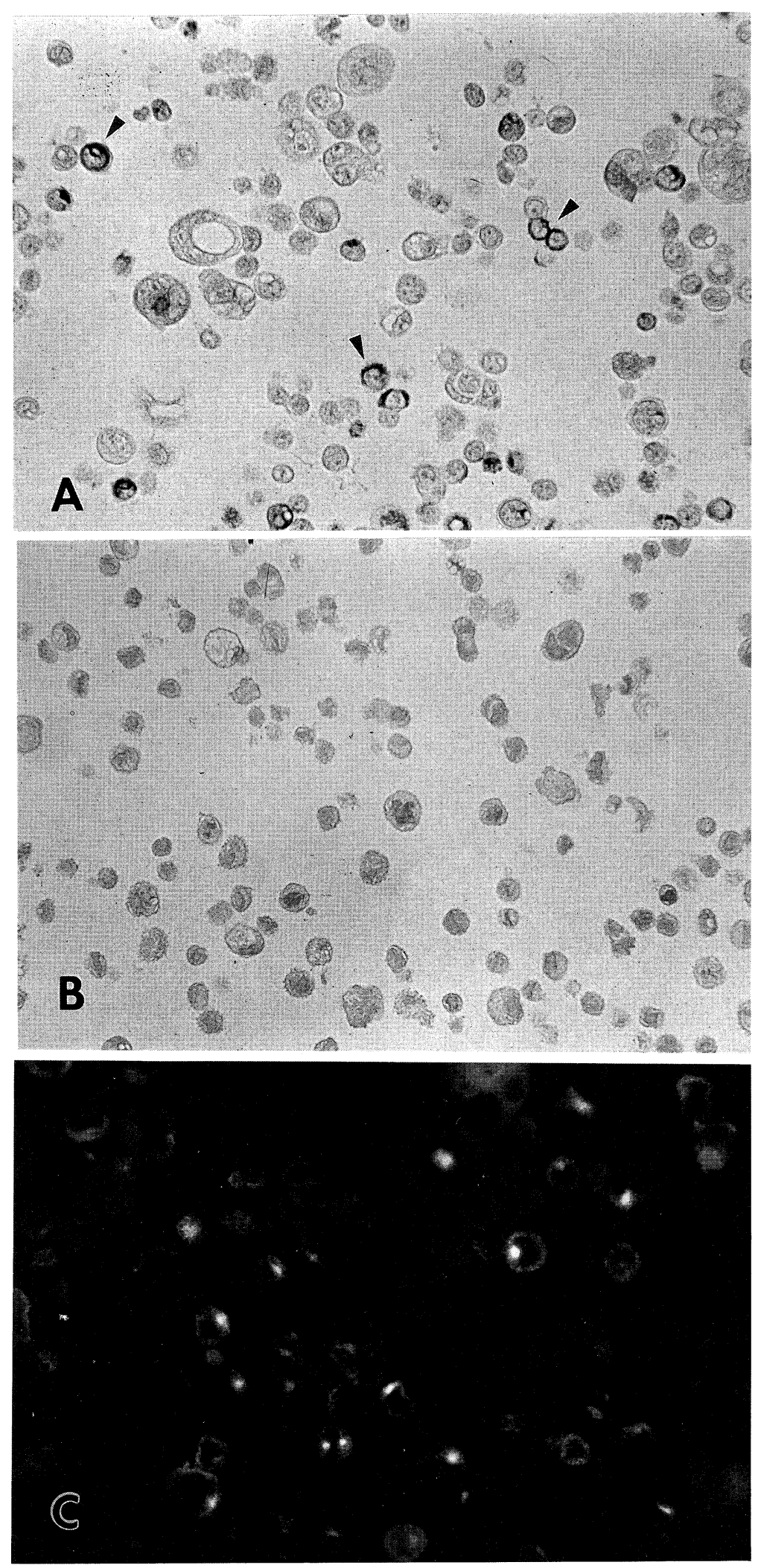

Fig. 1. Indirect immunofluorescence and PAP-staining microscopy with anti-villin, anti-ezrin, and antiMVM antibodies on paraffin sections of KATO-III cells. A: PAP-staining with anti-villin antibody $(200 \times)$. B: PAP-staining with anti-ezrin antibody $(200 \times)$. C: Immunofluorescence with anti-MVM antibody and FITC-conjugated secondary antibody $(200 \times)$. The cell surfaces are densely stained with villin $\mathrm{mAb}$ (arrowheads in A), and some labeled spots can be seen in the cytoplasm (C) whereas no positive staining was seen with anti-ezrin antibody (B). 

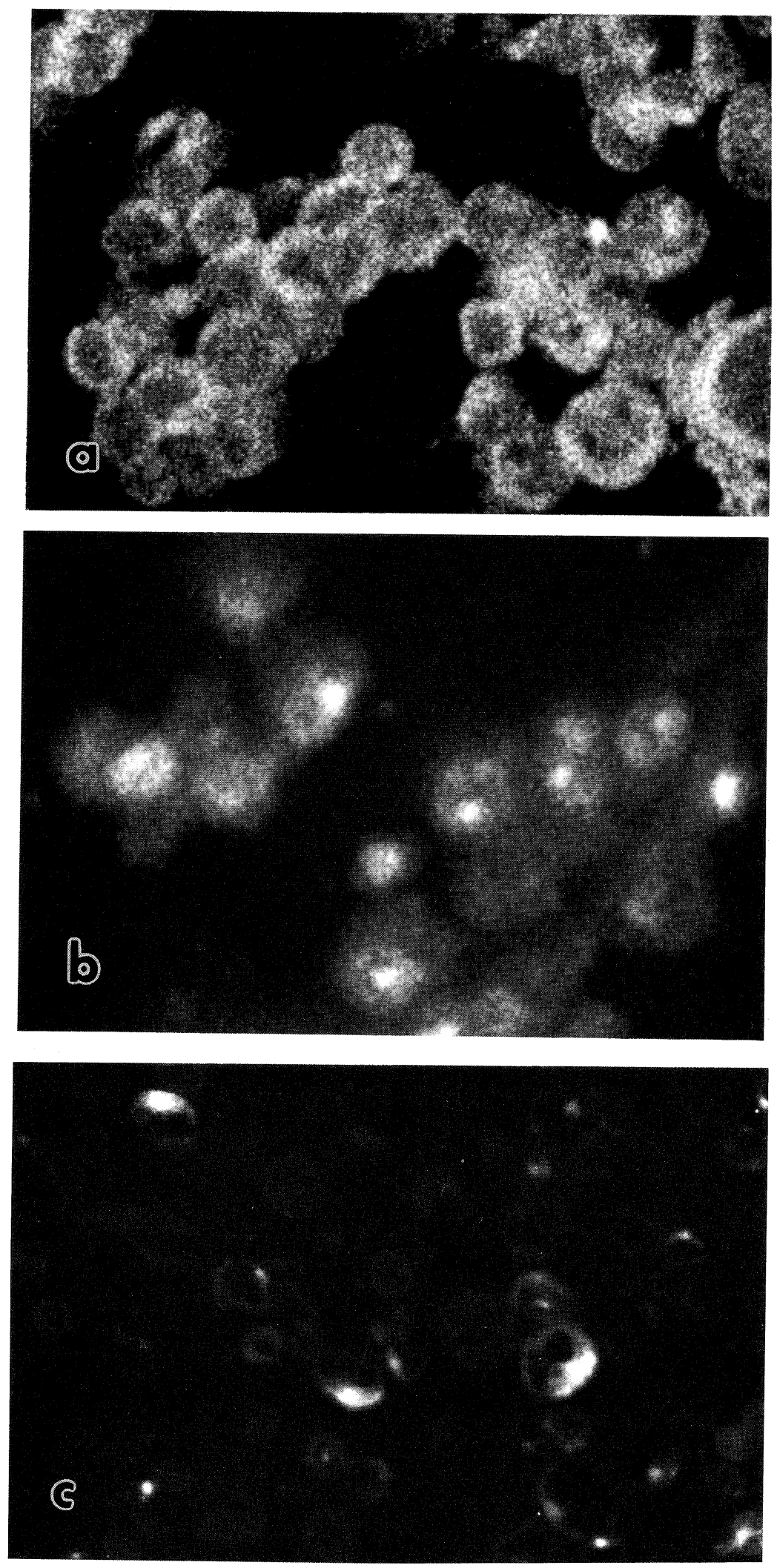

Fig. 2. Confocal analysis of immunofluorescence staining of the KATO-III cells with anti-villin and anti-MVM antibodies. Fluorescence images of the cells with anti-villin antibody and FITC-conjugated secondary antibody $(a, b)$. Staining can be seen densely and uniformly over the cell surface $(\mathrm{a}, 600 \times)$, and intracellular fluorescence staining was shown (b, $600 \times)$. Immunofluorescence staining of the cells with anti-MVM shows patch or "cap-like" spots at the cell surface $(c, 600 \times)$. 
times. The cells were post-fixed by resuspension in $0.5 \%$ $\mathrm{OsO}_{4}$. The samples were subjected to a graded series of alcohol and propylene oxide and embedded in Epon. Thin sections obtained with an ultramicrotome were post-stained with uranyl acetate and lead citrate.

Cell fractionation and subfractionation. KATO-III cells (about $1 \times 10^{7}$ cells) were homogenized in $2.5 \mathrm{mM}$ imidazole$\mathrm{HCl}$ buffer, $\mathrm{pH} 7.4$, containing $8 \%$ sucrose, $1 \mathrm{mM}$ PMSF, and $1 \mathrm{mM}$ NEM on ice with a Silverson homogenizer. The homogenate was fractionated as described previously (10). The microsomal fraction was loaded on a $41 \%$ solution of sucrose in 0.01 M Tris-EDTA (TE) buffer $\mathrm{pH}$ 7.4. After centrifugation at $100,000 \mathrm{~g}$ for 2 hours in a Swing rotor (Beckman), cell membranes were collected as a white interfacial band. The membrane fraction was diluted by adding a 3-4 fold excess of TE buffer $\mathrm{pH} 7.4$ and pelleted by centrifugation at $100,000 \mathrm{~g}$ for $2 \mathrm{~h}$.

SDS-PAGE and Immunoblotting. Samples were applied to SDS-polyacrylamide gel electrophoresis (PAGE) according to Laemmli (14). After electrophoresis (5-15\% gradient gel), the proteins were electrophoretically transferred to a nitrocellulose membrane. The membrane filter was preincubated with $1 \% \mathrm{BSA} / \mathrm{PBS}$ for $30 \mathrm{~min}$ and then incubated with a $1 / 500 \sim 1 / 200$ dilution of the primary antibodies in $1 \% \mathrm{BSA} /$ PBS buffer, pH 7.4, for $45 \mathrm{~min}$. Antigens were detected with peroxidase-conjugated goat anti-rabbit IgG or anti-mouse
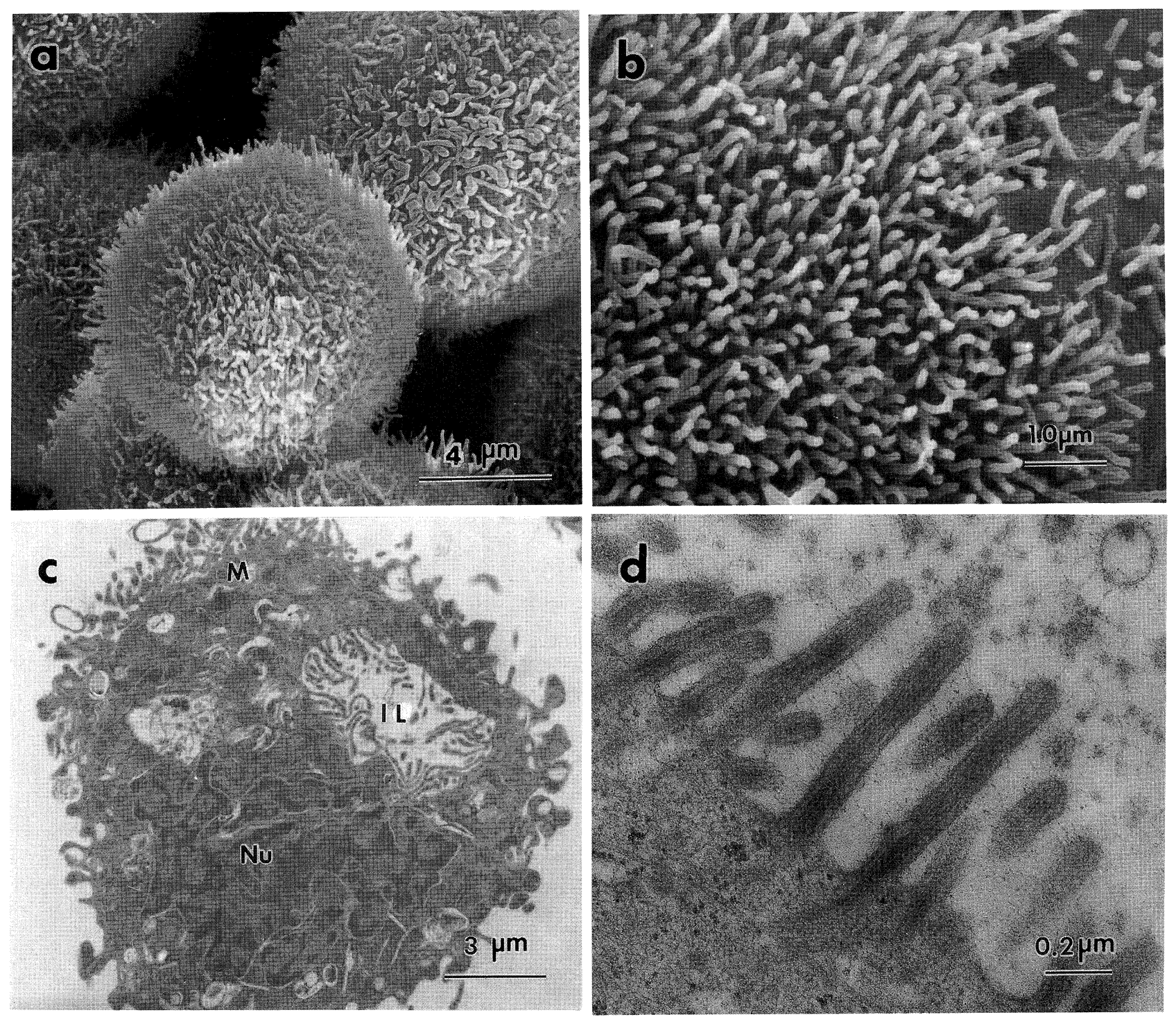

Fig. 3. Fine structures of the KATO-III cell. a, b: SEM shows numerous microvilli on the cell surface. c: TEM shows intracellular lumen with brush border-like structure (IL), nucleus (Nu), and surface microvilli (M). d: Higher magnification of the surface microvilli (d). Actin filaments are seen in the core and at the rootlet of the microvilli. 
IgG at a dilution of $1 / 1000$ (Cappel).

\section{RESULTS}

Immunostaining. The cells were harvested after one week of culture, and fixed with methacaln at $4^{\circ} \mathrm{C}$ overnight as described in Methods. The cells were then embedded in paraffin. Immunofluorescence- or PAP-staining of KATO-III cells treated with the monoclonal antivillin, anti-ezrin and polyclonal anti-MVM antibodies were shown in Fig. 1. Not only cell surface membranes but also some intracellular structures stained with antivillin but not anti-ezrin antibody. Anti-MVM antibody also reacted with the cells strongly (Fig. 1-c). The numerous microvilli of the cell surface were extensively stained with the antibody and some positive intracellular inclusion was shown by laser confocal microscopy after treating the cell with $0.5 \%$ Triton $\mathrm{X}-100$ for $5 \mathrm{~min}$ (Fig. 2).

Because methyl green and janus green distinguished
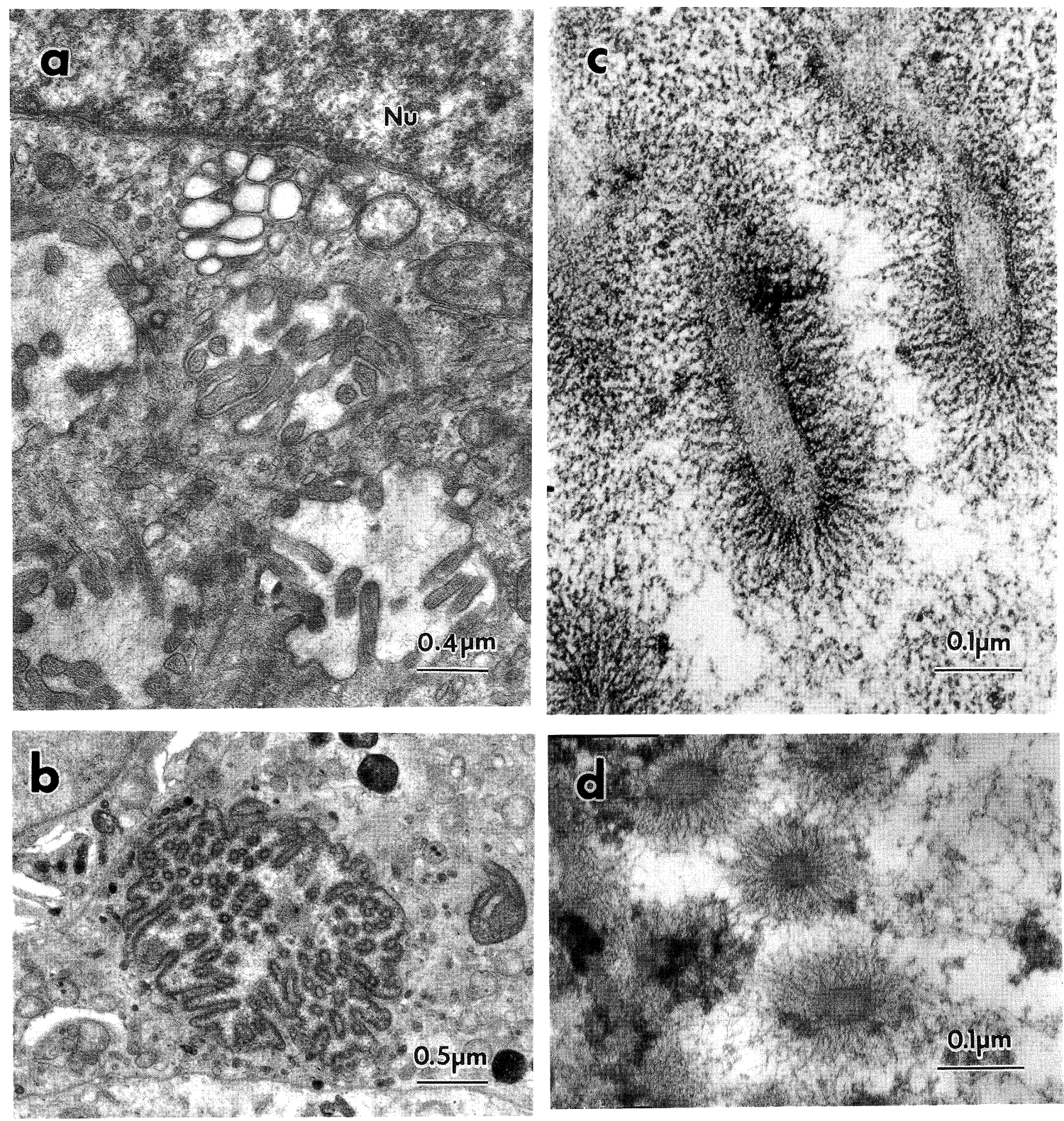

Fig. 4. Ultrastructures of microvilli of KATO-III cell. Microvillous inclusions are observed in the cell (a, b). Higher magnification revealed numerous glycocalyx over the microvilli (c, d). Some cross-sections of microvilli are shown (d). Actin filament cores are observed in the microvilli (c). 


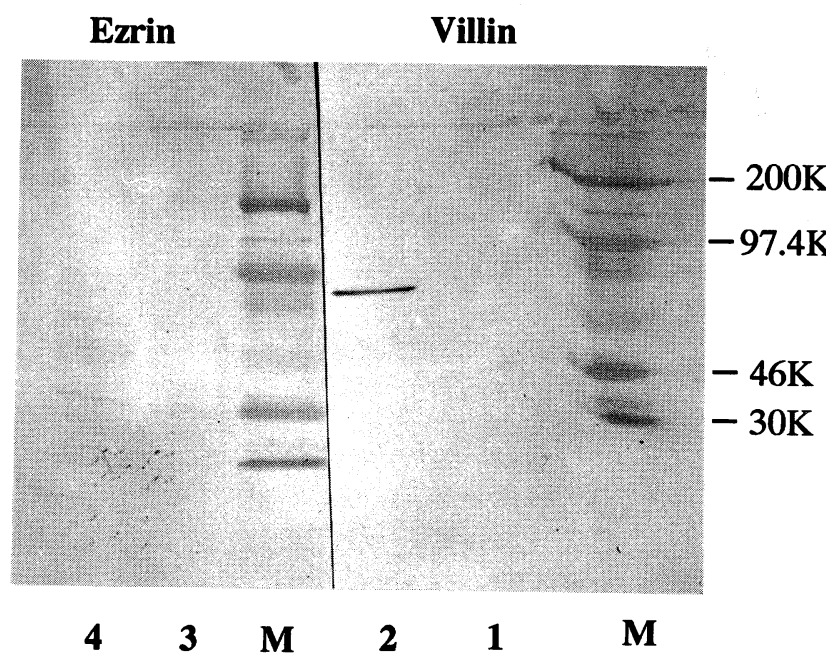

Fig. 5. Identification of the antigens of KATO-III cells recognized by the anti-villin and anti-ezrin antibody on nitrocellulose membrane. Details are described in Methods section. Samples were obtained by subcellular fractionation of the cells and Western blotting. Figure shows post-microsomal supernatant (lane 1,3), membranous fraction (lane 2, 4, fractions of interface of $41 \%$ sucrose), and Mr markers (M). Staining of anti-villin and anti-ezrin antibodies are lanes 1, 2 and lane 3,4 , respectively. Note that only one polypeptide of about $90 \mathrm{k}$ is detected using anti-villin antibody while no detectable band is seen using anti-ezrin antibody.

these cellular organella (data not shown), the intracellular staining by the antibodies was not due to nuclei and mitochondria.

Ultrastructure of the microvilli in KATO-III cells. The morphological study of KATO-III cells was performed using scanning electron microscopy (SEM) and transmission electron microscopy (TEM), which revealed cell surfaces covered with numerous microvilli (Fig. 3-a, b). Numerous cell surface microvilli varied in length and shape. By TEM analysis and intracellular lumen was observed (Fig. 3-c). The outer cell surface microvilli consisted of actin filament cores (Fig. 3-d). As shown in Fig. 4, some intracellular cysts lined with brush border-like structures were also observed. The size of the intracellular microcyst ranged from 0.6 to 4 $\mu \mathrm{m}$ in diameter. A glycocalyx-like layer was observed on the surface of the microvilli of the intracellular cyst (Fig. 4-b, c, d), and core bundles of microvilli as actin filaments of intracellular microcysts resembled those of cell surface microvilli (Fig. 3-d, Fig. 4-a).

Antigen distribution in subcellular fractions of KATO-III cell. The cell homogenate was cell fractionated, and the microsomal pellet was subfractionated as described in Methods. Each fraction was electrophoresed on SDS-polyacrylamide gel and analyzed for antivillin, anti-ezrin and anti-MVM antibodies by Western blotting as described in Methods. All immunoreactiv-

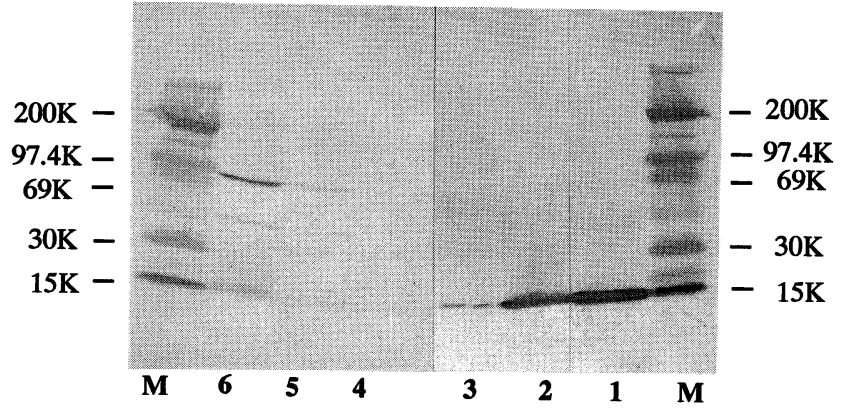

Fig. 6. Distribution of villin and antigens of anti-MVM antibody in KATO-III cell fractionation. Western blot analysis was carried out after SDS-PAGE (5-15\% gradient gel) of each subcellular fraction. The nitrocellulose filters were reacted with anti-MVM (lanes 1, 2, 3) and with anti-villin (lane 4, 5, 6). Figure shows membranous fractions (lanes 1, 6), cell homogenate (lanes 2, 5), post-microsomal supernatant (lanes 3, 4), and $\mathrm{Mr}$ marker standards (M). The $95 \mathrm{~K}$ and $15 \mathrm{~K}$ bands were detected with anti-villin and anti-MVM antibodies, respectively. Some nonspecific bands were seen at low molecular weight (lane 6).

ities on nitrocellulose after transfer from SDS-PAGE gel were shown at the 90-95 K molecular weight corresponding to villin (Fig. 5-lane 2, Fig. 6-lanes 5, 6). The highest immunoreactivity was detected in the membranous fraction of interface of $41 \%$ sucrose. The postmicrosomal supernatant did not contain villin antigen. Ezrin was detected neither in microsome nor supernatant (Fig. 5-lane 3, 4). In the case of anti-MVM antibody, the antigen of $15 \mathrm{k}$ protein was detected on a nitrocellulose after immunoblotting (Fig. 6-1, 2, 3). The electrophoretic pattern of the protein was not affected by treatment with disulfide-reducing agent with or without urea prior to electrophoresis (data not shown).

\section{DISCUSSION}

In the present study microvillous cytoskeletal components of the KATO-III cell were demonstrated by indirect immunostaining with an anti-villin and antiMVM antibodies but not ezrin-antibody. Villin, one of cytoskeletal parts of the microvillus, has been used as a marker of epithelial carcinogenesis $(12,17,18)$. Osborn et al. (18) demonstrated villin in signet ring cell carcinomas which were also aminopeptidase $\mathrm{N}$ and sucrase-isomaltase-positive. However, ezrin, one of cytoskeletons of intestinal microvilli (6), was not detected in KATOIII cell immunocytochemically or by Western blot analysis of cell homogenate and microsome in this study. As positive control for anti-ezrin antibody, we used the human cell lines such as Intestine 407 and OS-RC-2, obtained from Riken Cell Bank (Japan). It has been established that the monoclonal antibody reacted positively with these cells immunocytochemically and $80 \mathrm{~K}$ protein was found by Western blot analysis (data not 
shown).

The cytoplasmic distribution of villin-immunoreactivity has been reported for some colon adenocarcinomas $(17,21)$. Because ultrastructural study was not performed in those works it was unknown whether the intracellular villin-immunoreactivity was due to brush border-like structures or villin molecules dispersed in the cytoplasm. The intracellular microcysts immunostained with anti-villin antibody in the present work might correspond to the intracellular lumina observed by TEM. However, villin detected in the membranous fraction did not mean that it was of intracellular membrane origin because it is well known that fragmented microvilli are recovered in microsomal fractions (4). Cytoplasmic villin-immunoreactivity was demonstrated for some colon carcinomas $(17,21)$ and for HT-29 cells (4). Numerous microvilli have been found distributed throughout the cytoplasm of the parietal cell. The formation of microvilli in a stimulated condition was regulated by several cytoskeletal proteins including ezrin $(10,14,16)$. The existence of microvilli of intracellular structures in the KATO-III cells are similar to that of these cells, although ezrin was not detected.

In the case of anti-MVM antibody, the positive reaction with the KATO-III cell indicated the existence of similar antigenicity between intestinal microvilli and the cultured cell. There are two types of of KATO-III cells, one producing mucin and the other forming intracellular microcysts. The $15 \mathrm{KDa}$ protein was negative for PAS-staining indicating that the protein was not intensively glycosylated (data not shown). In agreement with this fact, anti-MVM immunostaining did not parallel PAS-staining in KATO-III cells. Little is known about $15 \mathrm{KDa}$ protein of KATO-III cell, but when the polypeptide was subjected to microsequencing analysis after being transferred onto PVDF membrane, it resulted in sequence homology to profilin protein which was one of actin-binding proteins (data not shown). Thus, it is suggested that several actin-binding proteins was related to the constitution of the KATO-III cell surface microvilli and intracellular microvilous inclusion.

Acknowledgements. We thank Ms. C. Yamashita and Mr. H. Igarashi for their valuable technical assistance.

\section{REFERENCES}

1. Arruda, M.V., Watson, S., Shwunlin, C., Leavitt, J., and Matsudaira, P. 1990. Fimbrin is a homologue of the cytoplasmic phosphoprotein plastin and has domains homologous with calmodulin and actin gelation proteins. J. Cell Biol., 111: 10691079.

2. BACCHI, C.E. and Gown, A.M. 1991. Distribution and pattern of expression of villin, a gastrointestinal-associated cytoskeletal protein, in human carcinomas; a study employing paraffin-embedded tissue. Labo. Invest., 64: 418-424.
3. Berryman, M., Franck, Z., and Bretscher, A. 1993. Ezrin is concentrated in the apical microvilli of a wide variety of epithelial cells whereas moesin is found primarily in endothelial cells. J. Cell Science, 105: 1025-1043.

4. Borgstrom, M. and Dahlevist, A. 1958. Cellular localization solubilization and separation of intestinal glycosidases. Acta Chem. Scand., 12: 1997-2006.

5. Bretscher, A., Osborn, M., Wehland, J., and Weber, K. 1981. Villin associates with specific microfilamentous structures as seen by immunofluorescence microscopy on tissue sections and cells microinjected with villin. Exp. Cell Res., 135: 213-219.

6. BRETSCHER, A. 1983. Purification of an 80,000-dalton protein that is a component of the isolated microvillus cytoskeleton, and its localization in nonmuscle cells. J. Cell Biol., 97: 425432.

7. Bretscher, A. 1986. Purification of the intestinal microvillous cytoskeletal proteins villin, fimbrin, and ezrin. Methods in Enzymology, 134: 24-37.

8. Chantret, I., Barbat, A., Dussaulx, E., Brattain, M.G., and ZweIBAUM, A. 1988. Epithelial polarity, villin expression, and enterocytic differentiation of cultured human colon carcinoma cells: A survey of twenty cell lines. Cancer Res., 48: 19361942.

9. Dudouet, B., Robine, S., Huet, C., Sahuquillo-Merino, C., Blair, L., Coudrier, E., and Louvard, D. 1987. Changes in villin synthesis and subcellular distribution during intestinal differentiation of HT29-18 clones. J. Cell Biol., 105: 359-369.

10. Fujita, M., Ohta, H., Kawai, K., Matsui, H., and NaKao, M. 1972. Differential isolation of microvilli and basolateral plasma membranes from intestinal mucosa. Biochim. Biophys. Acta, 274: 336-347.

11. Fujita, M., Ohta, H., and Uezato, T. 1981. Characterization of brush borders purified in iso-osmotic medium and microvillar membranes subfractionated from mouse small intestine. Biochem. J., 196: 669-673.

12. Gröne, H.J., Weber, K., Holmchen, U., and Osborn, M. 1986. Villin, a marker of brush border differentiation and cellular origin in human renal cell carcinoma. Am. J. Pathol., 124: 294-302.

13. Hanzel, D., Reggio, H., Bretscher, A., Forte, J.G., and MANGEAT, P. 1991. The secretion-stimulated $80 \mathrm{~K}$ phosphoprotein of parietal cells is ezrin, and has properties of a membrane cytoskeletal linker in the induced apical microvilli. EMBO, J., 10: $2363-2373$.

14. LAEMMLI, U.K. 1970. Cleavage of structural proteins during the assembly of the head of bacteriophage T4. Nature, 227: 680-685.

15. Lesuffleur, T., Barbat, A., Dussaulx, E., and Zweibaum, A. 1990. Growth adaptation to methotrexate of HT-29 human colon carcinoma cell is associated with their ability to differentiate into columnar absorptive and mucus-secreting cells. Cancer Res., 50: 6334-6343.

16. Mercier, F., Reggio, H., Devilliers, G., Bataille, D., and Mangeat, P. 1989. Membrane-cytoskeleton dynamics in rat parietal cells; mobilization of actin and spectrin upon stimulation of gastric acid secretion. J. Cell Biol., 108: 441-453.

17. Moll, R., Robine, B., Dudouet, B., and Louvard, D. 1987. Villin, a cytoskeletal protein and a differentiation marker expressed in some human adenocarcinomas. Virchows Arch B, 54: 155-169.

18. Osborn, M., Mazzoleni, G., Santini, D., Marrano, D., Martineli, G., and WeBer, K. 1988. Villin, intestinal brush 
border hydrolases and keratin polypeptides in intestinal metaplasia and gastric cancer; an immunohistologic study emphasizing the different degrees of intestinal and gastric differentiation in signet ring cell carcinomas. Virchows Arch A, 413: 303-312.

19. Robine, S., Huet, C., Moll, R., Sahuquillo-Merino, C., Coudrier, E., Zweibaum, A., and Louvard, D. 1985. Can villin be used to identify malignant and undifferentiated normal digestive epithelial cells? Proc. Natl. Acad. Sci. U.S.A., 82: 8488-8492.

20. Sekiguchi, M., Sakakibara, K., and FujiI, G. 1978. Establishment of cultured cell lines derived from a human gastric carcinoma. Jpn. J. Exp. Med., 48: 61-68.
21. West, A.B., Isaac, C.A., Carboni, J.M., Morrow, J.S., MoOSEKer, M.S., and BARWICK, K.W. 1988. Localization of villin, a cytoskeletal protein specific to microvilli, in human ileum and colon and in colonic neoplasms. Gastroenterology, 94: 343-352.

22. Yamabayashi, S., Fuji, Y., Kawahara, I., Iida, F., Ohno, S., and Nagata, T. 1979. Electron microscopic observation of the cultured human gastric carcinoma cells, an established cell line KATO-III. J. Clin. Electron Microscopy, 12: 5-6.

(Received for publication, April 29, 1995

and in revised form, May 25, 1995) 